\title{
Non-response to (statin) therapy: the importance of distinguishing non-responders from non-adherers in pharmacogenetic studies
}

\author{
S. Trompet ${ }^{1,2}$ - I. Postmus $^{2}$ - P. E. Slagboom ${ }^{3}$ - B. T. Heijmans ${ }^{3}$ - R. A. J. Smit ${ }^{1,2}$. \\ A. B. Maier ${ }^{4}$ - B. M. Buckley ${ }^{5}$ - N. Sattar ${ }^{6}$ - D. J. Stott ${ }^{7}$ - I. Ford ${ }^{8}$. \\ R. G. J. Westendorp ${ }^{9}$ - A. J. M. de Craen ${ }^{2}$. J. W. Jukema ${ }^{1,10,11}$
}

Received: 1 July 2015 / Accepted: 1 December 2015 / Published online: 19 December 2015

(C) The Author(s) 2015. This article is published with open access at Springerlink.com

\begin{abstract}
Purpose In pharmacogenetic research, genetic variation in non-responders and high responders is compared with the aim to identify the genetic loci responsible for this variation in response. However, an important question is whether the non-responders are truly biologically non-responsive or actually non-adherent? Therefore, the aim of this study was to describe, within the PROspective Study of Pravastatin in the Elderly at Risk (PROSPER), characteristics of both nonresponders and high responders of statin treatment in order to possibly discriminate non-responders from non-adherers. Methods Baseline characteristics of non-responders to statin therapy ( $\leq 10 \%$ LDL-C reduction) were compared with those of high responders ( $>40 \%$ LDL-C reduction) through a linear regression analysis. In addition, pharmacogenetic candidate gene analysis was performed to show the effect of excluding non-responders from the analysis.

Results Non-responders to statin therapy were younger $(p=0.001)$, more often smoked $(p<0.001)$, had a higher
\end{abstract}

S. Trompet

s.trompet@lumc.nl

1 Department of Cardiology, C5-R, Leiden University Medical Center, PO Box 9600, 2300 RC Leiden, The Netherlands

2 Department of Gerontology and Geriatrics, Leiden University Medical Center, Leiden, The Netherlands

3 Section of Molecular Epidemiology, Leiden University Medical Center, Leiden, The Netherlands

4 Section Gerontology and Geriatrics, Department of Internal Medicine, VU Medical Center, Amsterdam, The Netherlands

5 Department of Pharmacology and Therapeutics, University College Cork, Cork, Ireland alcohol consumption $(p<0.001)$, had lower LDL cholesterol levels $(p<0.001)$, had a lower prevalence of hypertension $(p<0.001)$, and had lower cognitive function $(p=0.035)$ compared to subjects who highly responded to pravastatin treatment. Moreover, excluding non-responders from pharmacogenetic studies yielded more robust results, as standard errors decreased.

Conclusion Our results suggest that non-responders to statin therapy are more likely to actually be non-adherers, since they have more characteristics that are viewed as indicators of high self-perceived health and low disease awareness, possibly making the subjects less adherent to study medication. We suggest that in pharmacogenetic research, extreme nonresponders should be excluded to overcome the problem that non-adherence is investigated instead of non-responsiveness.

Keywords Pharmacogenetics $\cdot$ Adherence $\cdot$ Statins · Cardiovascular
6 BHF Glasgow Cardiovascular Research Centre, Faculty of Medicine, Glasgow, UK

7 Institute of Cardiovascular and Medical Sciences, Faculty of Medicine, University of Glasgow, Glasgow, UK

8 Robertson Center for Biostatistics, University of Glasgow, Glasgow, UK

9 Faculty of Health and Medical Sciences, Department of Public Health, University of Copenhagen, Copenhagen, Denmark

10 Durrer Center for Cardiogenetic Research, Amsterdam, The Netherlands

11 Interuniversity Cardiology Institute of the Netherlands, Utrecht, the Netherlands 
Hydroxymethyl-3-methylglutaryl coenzyme A (HMG-CoA) reductase inhibitors (statins) are the most commonly prescribed drugs for the prevention of cardiovascular disease worldwide. Statins lower plasma cholesterol levels by 30 $50 \%$ and are associated with a reduction of cardiovascular events of $20-40 \%$ [1]. Statins are generally well tolerated and are believed to have relatively few side effects [2]. However, clinical response is highly variable and not all subjects appear to benefit from statin therapy, with roughly a third of treated patients achieving the lipid lowering goals specified in international guidelines [1]. It is uncertain whether these subjects are truly biologically unresponsive, or are actually nonadherent to the statin therapy.

Pharmacogenetic studies aim to find genetic variation that is responsible for the variable response to drug treatment. For that purpose, high responders and non-responders are often compared with the aim to identify genetic loci associated with the variation in response [ 3, 4]. Particularly in whole genome sequencing studies, only the two extreme phenotypes (i.e., the extremely good responders and the non-responders) are chosen to reduce costs and enhance efficiency [5]. However, for correct interpretation of this comparison, it is essential to ensure that non-responders have actually taken the drug and are not merely non-responders due to non-adherence.

The most suitable studies to investigate these pharmacogenetic effects are randomized controlled trials, since adherence to medication is closely monitored, for example, through questionnaires and pill count and increasingly through electronic medication monitoring devices [6]. However, these monitoring systems do not provide absolute certainty that subjects are actually adherent to their medication [7]. Non-adherers can relatively easily bypass the control mechanisms, e.g., by discarding drugs before the pill count, which may easily lead to overestimation of adherence [8]. Using data of the PROspective Study of Pravastatin in the Elderly at Risk (PROSPER) [9, 10], we describe baseline characteristics of differential responder groups to statin treatment in order to find discriminatory factors between biologically non-responders and likely non-adherers. Furthermore, we propose how to deal with the misclassification of "false" non-responders in pharmacogenetic analyses.

\section{Methods}

We used data from the PROSPER study [ 9, 10]. In short, the PROSPER study is a prospective multicenter randomized placebo-controlled trial to assess whether treatment with $40 \mathrm{mg}$ daily pravastatin diminishes the risk of major vascular events in elderly individuals. Men and women aged 70 82 years were recruited if they had pre-existing vascular disease or were at increased risk of such disease due to smoking, hypertension, or diabetes. A total number of 5804 subjects were randomly assigned to pravastatin $(n=2891)$ or placebo treatment $(n=2913)$. At baseline, a brief medical history was taken, vital signs were recorded, and a fasting venous blood sample was collected for biochemical and hematological checks and for lipoprotein quantification. In addition, a mini-mental state examination (MMSE) was conducted to test for cognitive function, and subjects with an MMSE score of 24 or lower were excluded. The protocol of the PROSPER study meets the criteria of the Declaration of Helsinki and was approved by the medical ethics committees of each participating institution. Written informed consent was obtained from all participating cohorts.

From the pravastatin users $(n=2891)$, we excluded all subjects who were withdrawn from the PROSPER study during follow-up due to refusal of study medication or did not attend the follow-up visits $(n=346)$. For the remaining subjects $(n=2545)$, response to statin treatment was defined as the percentage of achieved LDL lowering. This was calculated by dividing the difference between baseline and post-baseline (mean of measurements at months 3, 6, 12, 24, and 36) LDL levels by the baseline level and multiplying the result by 100 . If data of one or more of the measurements for one individual was missing, we took the mean of the available measurements of that individual as post-statin treatment measurement. These data were available for 2519 subjects.

We then created five groups of achieved LDL lowering $(\leq 10,10-20,20-30,30-40,>40 \%$ LDL lowering) and compared baseline characteristics between these groups. Based on clinical experience, non-responders were defined as those subjects achieving a $10 \%$ or lower decrease in LDL cholesterol levels and high responders were defined as those subjects achieving a decrease of more than $40 \%$.

First, we assessed whether there were differences in baseline characteristics between the five groups of achieved LDL lowering using ANOVA. Baseline characteristics included sex, age, education, smoking, alcohol use, BMI, blood pressure, LDL cholesterol level, history of hypertension, diabetes, vascular disease, and cognitive function. We also assessed differences in baseline characteristics between the high and non-responders with the Student's $t$ test for continuous variables and the Pearson chi-square test for categorical variables.

Second, we used binary logistic regression to assess the relative risk of being a non-responder based on the clinical characteristics that were significantly different between the high and low groups in the first analysis. Continuous measurements were dichotomized based on sex-specific medians. All analyses were adjusted for age and country of origin, and where necessary additionally adjusted for sex. Third, we calculated the number of risk factors per subject and assessed the association between the number of risk factors and nonresponder status with binary logistic regression analysis 
adjusted for age, sex, and country of origin. We were unable to calculate the sum of risk scores in seven subjects, six of whom were high responders, due to missing data.

Finally, we performed a pharmacogenetic candidate gene study to show the effect of four well-established associated SNPs with a variable response to statin therapy using four analytic strategies [11]. The four SNPs (rs2900478 (SLCO1B1), rs445925 (APOE), rs464776 (SORT1/ CELSR2/PSRC1), and (rs10455872 (LPA) were extracted from the Genome Wide Association Study (GWAS) performed in the PROSPER study, named the PHASE study (the PHArmacogenetic study of Statins in the Elderly) [12]. Genotyping was performed with the Illumina $660 \mathrm{~K}$ beadchip, and imputation of up to 2.5 million SNPs was based on the HapMap 36 build. First, we performed a linear regression analysis to investigate the effect of the four SNPs on the achieved LDL lowering (\%) in the total study sample $(n=2272)$ adjusted for age, sex, country, and baseline LDL levels. The total number of subjects is lower in this genetic analysis since the GWAS has not been executed in all
PROSPER subjects, because of genotyping failure or because subjects were excluded based on the GWAS quality control criteria [12]. No subjects were excluded based on phenotypic outliers. Second, we excluded the non-responders from the sample and repeated the linear regression analysis $(n=2167)$. Third, we compared the effect of the SNPs in a case-control setting where the non-responders were set as the cases and the high responders as controls by using binary logistic regression adjusted for age, sex, country, and baseline LDL levels $(n=669)$. Lastly, we performed the same analysis using the low to moderate responders (10-20\% achieved LDL lowering) as cases $(n=817)$.

\section{Results}

Table 1 shows the baseline characteristics of the five groups of percentage LDL lowering with pravastatin treatment. There were significant differences between the groups with regard to sex, smoking status, history of hypertension, age,
Table 1 Association between groups of \% LDL lowering to statin treatment and clinical variables

\begin{tabular}{|c|c|c|c|c|c|c|}
\hline & \multicolumn{5}{|c|}{$\%$ LDL lowering in response to pravastatin treatment } & \multirow[b]{2}{*}{$\begin{array}{l}p \\
\text { ANOVA }\end{array}$} \\
\hline & $\begin{array}{l}>40 \% \\
(n=734)\end{array}$ & $\begin{array}{l}30-40 \% \\
(n=989)\end{array}$ & $\begin{array}{l}20-30 \% \\
(n=502)\end{array}$ & $\begin{array}{l}10-20 \% \\
(n=180)\end{array}$ & $\begin{array}{l}\leq 10 \% \\
(n=114)\end{array}$ & \\
\hline \multicolumn{7}{|l|}{$\begin{array}{l}\text { Categorical variables } \\
\quad(n, \%)\end{array}$} \\
\hline Females & $423(58)$ & $511(52)$ & $218(43)$ & $82(46)$ & $56(49)$ & $<0.001$ \\
\hline Current smokers & $126(17)$ & $244(25)$ & $151(30)$ & $65(36)$ & $54(47)^{*}$ & $<0.001$ \\
\hline $\begin{array}{l}\text { History of } \\
\text { hypertension }\end{array}$ & $503(69)$ & $620(63)$ & $301(60)$ & $98(54)$ & $58(51)^{*}$ & $<0.001$ \\
\hline History of diabetes & $79(11)$ & $104(11)$ & $58(12)$ & $16(8)$ & $7(6)$ & 0.485 \\
\hline $\begin{array}{l}\text { History of vascular } \\
\text { disease }\end{array}$ & $335(46)$ & $437(44)$ & $228(45)$ & $77(43)$ & $46(40)$ & 0.809 \\
\hline \multicolumn{7}{|l|}{ Country } \\
\hline Scotland & $325(44)$ & $410(42)$ & $210(42)$ & $83(46)$ & $49(43)$ & \\
\hline Ireland & $248(34)$ & $364(37)$ & $200(40)$ & $71(39)$ & $51(45)$ & \\
\hline The Netherlands & $161(22)$ & $215(22)$ & $92(18)$ & $26(14)$ & $14(12)$ & 0.253 \\
\hline \multicolumn{7}{|c|}{ Continuous variables (mean, SE) } \\
\hline Age (years) & $75.7(0.12)$ & $75.3(0.11)$ & $75.0(0.15)$ & $75.1(0.24)$ & $74.6(0.29)^{*}$ & 0.001 \\
\hline BMI $\left(\mathrm{kg} / \mathrm{m}^{2}\right)$ & $26.9(0.15)$ & $26.8(0.13)$ & $26.9(0.18)$ & $27.1(0.33)$ & $26.3(0.42)$ & 0.433 \\
\hline Education (years) & $15.2(0.08)$ & $15.3(0.07)$ & $15.3(0.10)$ & $14.5(0.11)$ & $15.2(0.19)$ & $<0.001$ \\
\hline MMSE (points) & $28.1(0.06)$ & $28.2(0.05)$ & $28.0(0.07)$ & $27.8(0.12)$ & $27.8(0.14)^{*}$ & 0.010 \\
\hline $\begin{array}{l}\text { Alcohol } \\
\quad \text { (units/week) }\end{array}$ & $3.5(0.29)$ & $5.0(0.27)$ & $7.2(0.47)$ & $7.2(0.80)$ & $6.5(0.90)^{*}$ & $<0.001$ \\
\hline $\begin{array}{l}\text { LDL cholesterol (mmol/ } \\
\text { L) }\end{array}$ & $4.0(0.03)$ & $3.8(0.02)$ & $3.7(0.03)$ & $3.4(0.05)$ & $3.4(0.08)^{*}$ & $<0.001$ \\
\hline $\mathrm{SBP}(\mathrm{mmHg})$ & $\begin{array}{c}156.0 \\
(0.80)\end{array}$ & $\begin{array}{l}154.0 \\
(0.70)\end{array}$ & $\begin{array}{c}155.8 \\
(0.99)\end{array}$ & $\begin{array}{l}153.4 \\
(1.59)\end{array}$ & $152.8(2.14)$ & 0.200 \\
\hline DBP (mmHg) & $83.8(0.41)$ & $83.6(0.36)$ & $83.6(0.50)$ & $82.7(0.83)$ & $83.7(1.04)$ & 0.828 \\
\hline
\end{tabular}

$B M I$ body mass index, $M M S E$ mini-mental state examination, $L D L$ low-density lipoprotein, $S B P$ systolic blood pressure, $D B P$ diastolic blood pressure

*Significant difference between the groups of $\leq 10 \%$ and $>40 \%$ LDL lowering (all $p<0.05$ ) 
education, cognitive function, alcohol use, and level of LDL cholesterol. For most characteristics there was a linear trend with LDL lowering response, for alcohol use, we observed a plateau effect for LDL lowering of $30 \%$ and lower. Moreover, when we compared the baseline characteristics of the 114 non-responders with the characteristics of the 734 high responders to pravastatin therapy, we found that subjects who did not respond to pravastatin therapy were on average 1 year younger $(p=0.001)$, more often smoked and drank more alcohol (both $p<0.001$ ), had lower LDL cholesterol levels $(p<0.001)$, had lower prevalence of hypertension $(p<0.001)$, and had lower cognitive function $(p=0.035)$, compared to subjects who highly responded to pravastatin therapy. Based on pill count, we defined a nonadherer if they returned more than $18(20 \%)$ pills in the preceding 90 days before their study visit (mean pill count over maximum number of study visits per individual). Within the subjects that highly responded to pravastatin therapy, $99.5 \%$ were adherent to their study medication based on pill count, whereas in the non-responders group this was reduced to $78.6 \%$.

Next, we calculated the relative risk of being a nonresponder for the characteristics that significantly differed between high and non-responders with a binary logistic regression model (Table 2). The largest relative risk was found for subjects who were current smokers (OR 3.96, $95 \%$ CI 2.60$\left.6.03, p=1.4 \times 10^{-10}\right)$. We also found a higher risk of being a non-responder in subjects without a history of hypertension (OR 2.01, $95 \%$ CI 1.32-3.04, $p=0.001$ ), with a lower cognitive function (OR 1.46, $95 \%$ CI $0.97-2.20, p=0.068$ ), with higher alcohol intake (OR 1.73, $95 \%$ CI 1.15-2.59, $p=0.008$ ), and with lower LDL cholesterol levels (OR 3.14,
$95 \%$ CI $2.05-4.80, p=1.3 \times 10^{-7}$ ). The association between number of characteristics and response status is also shown in Table 2. Compared to subjects with none or one risk factor, the relative risk of being a non-responder gradually increased to $15.51\left(95 \%\right.$ CI 5.83-41.27, $p=4.0 \times 10^{-8}$ ) for subjects with five characteristics. When the summary score was included in the model as a continuous variable, the risk of being a nonresponder increased with 2.04 (95\% CI 1.69-2.46, $p=1.1 \times 10^{-13}$ ) per additional characteristic.

We examined the association of four well-established SNPs involved in pharmacogenetics of statins using four different research strategies (Table 3). By comparing the results for analyses 1 and 2 where we investigated the effect of the four SNPs with a continuous measurement of achieved LDL lowering (\%), it is shown that by exclusion of the non-responders to statin therapy, the standard errors (SE) decreases, indicating that indeed probably noise is removed from these analyses. The beta stays more or less consistent in the analyses; however, since the SE decreases, also the $p$ value decreases, and all four SNPs show significant results. In the third analysis, we compared the effect of the four SNPs in a case-control setting, where the non-responders were set as the cases and the high responders as controls, by using binary logistic regression adjusted for age, sex, country, and baseline LDL levels. Surprisingly, none of the four SNPs showed a significant association with statin response as was shown by the continuous analysis in the first two research strategies. However in analysis 4, we investigated the effect of the four SNPs also in a case-control setting, by comparing the high responders (controls) with the low-moderate responders (cases), thereby again excluding the non-responders.
Table 2 Association between baseline characteristics and being a non-responder

\begin{tabular}{lllll}
\hline & $\begin{array}{l}\text { High responders } \\
(n=734)\end{array}$ & $\begin{array}{l}\text { Non-responders } \\
(n=114)\end{array}$ & $\begin{array}{l}\text { OR } \\
(95 \% \mathrm{CI})^{\mathrm{a}}\end{array}$ & $p$ value \\
\hline Smoking & $126(17)$ & $54(47)$ & $3.96(2.60-6.03)$ & $1.43 \times 10^{-10}$ \\
No history of hypertension & $231(32)$ & $56(49)$ & $2.01(1.32-3.04)$ & 0.001 \\
Low MMSE & $379(52)$ & $68(60)$ & $1.46(0.97-2.20)$ & 0.068 \\
High alcohol & $270(37)$ & $58(51)$ & $1.73(1.15-2.59)$ & 0.008 \\
Low LDL cholesterol & $298(41)$ & $78(68)$ & $3.14(2.05-4.80)$ & $1.31 \times 10^{-7}$ \\
Number of characteristics & & & & \\
$\leq 1$ & $297(41)$ & $20(18)$ & $1.0(\mathrm{ref})$ & - \\
2 & $256(35)$ & $26(23)$ & $1.71(0.93-3.15)$ & 0.086 \\
3 & $126(17)$ & $36(32)$ & $4.77(2.63-8.63)$ & $2.56 \times 10^{-7}$ \\
4 & $38(5)$ & $20(18)$ & $7.26(3.47-15.19)$ & $1.43 \times 10^{-7}$ \\
5 & $10(1)$ & $11(10)$ & $15.51(5.83-41.27)$ & $3.97 \times 10^{-8}$ \\
Trend & & $2.04(1.69-2.46)$ & $1.13 \times 10^{-13}$ \\
\hline
\end{tabular}

$O R$ odds ratio, $L D L$ low-density lipoprotein, $S B P$ systolic blood pressure, MMSE mini-mental state examination

${ }^{a}$ The OR represents the risk of being a non-responder when you are in the risk category. The continuous factors were dichotomized based on sex-specific medians. Adjusted for age and country, the analyses for smoking and hypertension were additionally adjusted for sex 
Table 3 Comparison of four genetic association analyses with four well-known SNPs associated with a pharmacogenetic effect of statin therapy

\begin{tabular}{lcccc}
\hline & Number & Beta & SE & $p$ value \\
\hline rs2900478 (SLCO1B1) & & & & \\
1. All subjects & 2272 & 0.021 & 0.0065 & 0.0014 \\
2. Excluding non-responders & 2167 & 0.020 & 0.0061 & 0.0008 \\
3. High vs non-responders & 669 & -0.129 & 0.201 & 0.5209 \\
4. High vs low-moderate responders & 817 & -0.682 & 0.158 & 0.0001 \\
rs445925 (APOE) & & & & \\
1. All subjects & 2272 & -0.022 & 0.0088 & 0.0121 \\
2. Excluding non-responders & 2167 & -0.021 & 0.0082 & 0.0097 \\
3. High vs non-responders & 669 & 0.398 & 0.327 & 0.2236 \\
4. High vs low-moderate responders & 817 & 0.170 & 0.262 & 0.5165 \\
rs646776 (SORT1/CELSR2/PSRC1) & & & & \\
1. All subjects & 2272 & -0.014 & 0.0058 & 0.0129 \\
2. Excluding non-responders & 2167 & -0.016 & 0.0054 & 0.0033 \\
3. High vs non-responders & 669 & 0.060 & 0.179 & 0.7388 \\
4. High vs low-moderate responders & 817 & 0.358 & 0.162 & 0.0268 \\
rs10455872 (LPA) & & & & 0.0042 \\
1. All subjects & 2272 & 0.0351 & 0.0123 & 0.0122 \\
2. Excluding non-responders & 2167 & 0.0288 & 0.0115 & 0.0714 \\
3. High vs non-responders & 669 & -1.424 & 0.790 & 0.4372 \\
4. High vs low-moderate responders & 817 & -0.682 & 0.873 & \\
\hline
\end{tabular}

Analyses 1 and 2 were performed with linear regression with achieved LDL lowering (\%) as outcome, adjusted for age, sex, country, and baseline LDL levels. Analyses 3 and 4 were performed with binary logistic regression adjusted for age, sex, country, and baseline LDL levels
Now, two out of the four SNPs did again show a significant association with LDL response (Table 3).

\section{Discussion}

In this study, we show that non-responders to statin treatment differ from high responders with regard to baseline clinical characteristics. Non-responders were more likely to smoke, drank more alcohol, had a lower cognitive function, were less likely to have hypertension, and had lower LDL cholesterol levels. These characteristics can be considered as indicators of higher self-perceived health and lower disease awareness, indicating that non-responders are possibly less aware of the benefits of using the study medication and are therefore more likely to be non-adherent rather than biologically unresponsive. Moreover, we show that exclusion of the non-responders in pharmacogenetic analyses yields more robust results, as the standard errors decreased after exclusion and $p$ values remained significant. These results indicate that pharmacogenetic studies that compare extreme phenotypes might be at least partially biased by the phenomenon of some, perhaps many, non-adherers probably being misclassified as nonresponders.

Few studies have investigated differences between nonresponders and high responders of statin therapy [13-16].
These showed that characteristics that are indicators of better self-perceived health like age, the number of comorbidities, and diet habits are different between high and non-responders and are therefore more likely to be indicators of nonadherence $[17,18]$. However, we cannot rule out the possibility that these characteristics truly determine whether a subject responds biologically differently to statin therapy. For example, having higher LDL cholesterol baseline levels might be associated with greater response to statin treatment simply because a greater absolute, but also relative, change is possible, and also for example by making healthy lifestyle changes on top of statin treatment. Therefore it is still not certain whether this variable can help us to discriminate between non-responders and non-adherers. This argument holds also true for smoking and gender. For both characteristics, we can think of plausible reasons that these are related to the amount of adherence, but we cannot rule out the possibility of a true pharmacological effect between these characteristics and statin treatment. However, in various subgroup analyses within the PROSPER study and in the existing literature, we found no evidence of an interaction between any of the clinical characteristics and statin response [10].

To draw more definite conclusions whether a nonresponder is a true non-adherer, the plasma level of the medication should be determined. Unfortunately, we have not measured this, but even if we had, this would unfortunately 
not solve our problem since a patient could easily restart medication just prior to the plasma sample, while not taking the medication on other days. The second best is pill count, although also not considered highly reliable, especially not in willingly non-compliant (possibility of discarding pills just prior the pill count). Nevertheless we did keep track of the number of pills used by the participants by a provisional pill count. Based on pill count, we defined a non-adherer if they returned more than $18(20 \%)$ pills in the preceding 90 days before their study visit (mean pill count over maximum number of study visits per individual). Within the subjects that highly responded to pravastatin therapy, $99.5 \%$ were adherent to their study medication based on pill count, whereas in the non-responders group, this was reduced to $78.6 \%$. However, since multiple studies have shown that pill count is likely not a valid instrument to measure true adherence [7, 8], we cannot draw definite conclusions from these numbers.

In many pharmacogenetic studies, non-responders are compared to high responders to investigate which genetic variants are responsible for this difference in response [5]. Although we believe that by using this design both statistical power and efficiency are maximized, there is the possibility of unintentionally investigating the non-adherent phenotype. Hence, instead of finding genetic variation responsible for the variation in response to therapy, genetic variation for adherence is assessed. Therefore, we assessed the impact of four different research strategies while performing pharmacogenetic research. Our results suggest that by excluding non-responders, the noise of any possible non-adherence is reduced, as the standard errors decreased, which cannot be the result of a larger sample size. Moreover, through the use of a casecontrol design for optimal efficiency, we demonstrated that by using the non-responders as cases none of the four SNPs were significantly associated whereas by using low-moderate responders, two out of the four SNPs demonstrated a significant association.

Therefore, based on these results, our suggestion is that in pharmacogenetic research, instead of comparing the extreme phenotypes (high versus non-responders), other research strategies should be undertaken to find the genetic variation responsible for the difference in response to (statin) therapy. We propose three different strategies that may be followed to diminish the likelihood of investigating non-adherence instead of non-responsiveness. First, all subjects should be investigated using the full range of responsiveness as a continuous phenotype. This way, possible non-adherers among the extremely non-responsive cases will exert limited influence on the results. The second proposed strategy is to exclude nonresponsive subjects to be sure that the non-adherers are excluded from the analysis. The third, the most sophisticated, strategy is to use a propensity score, based on various clinical characteristics associated with non-adherence, to match high responders to non-responders when taking only the extremes into a pharmacogenetic study for efficiency. This last analysis will exclude any possible confounding from non-adherence from the study. Unfortunately, we could not perform such analysis in our data due to low statistical power.

In conclusion, pharmacogenetic studies that investigate the difference between high and non-responders are almost certainly in part investigating the non-adherent phenotype, since non-responders have clinical characteristics that coincide with high self-perceived health and low-disease awareness which are also very common in non-adherers. Other strategies, as proposed herein, should be used to investigate the relation between genetic variation and responsiveness to (statin) treatment.

Acknowledgments The research leading to these results has received funding from the European Union's Seventh Framework Programme (FP7/2007-2013) under grant agreement no. HEALTH-F2-2009223004. This work was performed as part of an ongoing collaboration of the PROSPER study group in the universities of Leiden, Glasgow, and Cork. Prof. Dr. J.W. Jukema is an established clinical investigator of The Netherlands Heart Foundation (2001 D 032).

Authors' contributions ST, AdC, and JWJ conceived the study. ST, IP, and RAJS performed and interpreted the statistical analysis. AdC, RGJW, and JWJ supervised the study. ST, IP, RAJS, AdC, and JWJ wrote the manuscript, and $\mathrm{BH}, \mathrm{PES}, \mathrm{DS}, \mathrm{NS}, \mathrm{ABM}, \mathrm{BMB}$, and IF critically revised the manuscript.

\section{Compliance with ethical standards}

Conflict of interest The authors declare that they have no competing interests.

Open Access This article is distributed under the terms of the Creative Commons Attribution 4.0 International License (http:// creativecommons.org/licenses/by/4.0/), which permits unrestricted use, distribution, and reproduction in any medium, provided you give appropriate credit to the original author(s) and the source, provide a link to the Creative Commons license, and indicate if changes were made.

\section{References}

1. Ballantyne CM (2004) Achieving greater reductions in cardiovascular risk: lessons from statin therapy on risk measures and risk reduction. Am Heart J 148(1 Suppl):S3-S8

2. Jukema JW, Cannon CP, de Craen AJ, Westendorp RG, Trompet S (2012) The controversies of statin therapy: weighing the evidence. J Am Coll Cardiol 60(10):875-881

3. Verschuren JJW, Trompet S, Wessels JA, Guchelaar HJ, Maat de MP, Simoons ML, et al. (2011) Pharmacogenetics in cardiovascular disease; ready for clinical application? European Heart Journal

4. Voora D, Ginsburg GS (2012) Clinical application of cardiovascular pharmacogenetics. J Am Coll Cardiol 60(1):9-20

5. Gurwitz D, McLeod HL (2013) Genome-wide studies in pharmacogenomics: harnessing the power of extreme phenotypes. Pharmacogenomics 14(4):337-339 
6. Farmer KC (1999) Methods for measuring and monitoring medication regimen adherence in clinical trials and clinical practice. Clin Ther 21(6):1074-1090

7. Osterberg L, Blaschke T (2005) Adherence to medication. N Engl J Med 353(5):487-497

8. Liu H, Golin CE, Miller LG, Hays RD, Beck CK, Sanandaji S, et al. (2001) A comparison study of multiple measures of adherence to HIV protease inhibitors. Ann Intern Med 134(10):968-977

9. Shepherd J, Blauw GJ, Murphy MB, Cobbe SM, Bollen EL, Buckley BM, et al. (1999) The design of a PROspective Study of Pravastatin in the Elderly at Risk (PROSPER). PROSPER study group. PROspective Study of Pravastatin in the Elderly at Risk. Am J Cardiol 84(10):1192-1197

10. Shepherd J, Blauw GJ, Murphy MB, Bollen EL, Buckley BM, Cobbe SM, et al. (2002) Pravastatin in elderly individuals at risk of vascular disease (PROSPER): a randomised controlled trial. Lancet 360(9346):1623-1630

11. Postmus I, Trompet S, Deshmukh HA, Barnes MR, Li X, Warren HR, et al. (2014) Pharmacogenetic meta-analysis of genome-wide association studies of LDL cholesterol response to statins. Nat Commun 5:5068

12. Trompet S, de Craen AJ, Postmus I, Ford I, Sattar N, Caslake M, et al. (2011) Replication of LDL GWAs hits in PROSPER/PHASE as validation for future (pharmaco)genetic analyses. BMC Med Genet $12: 131$

13. Cone C, Murata G, Myers O (2011) Demographic determinants of response to statin medications. Am J Health Syst Pharm 68(6):511517

14. Kim YS, Sunwoo S, Lee HR, Lee KM, Park YW, Shin HC, et al. (2002) Determinants of non-compliance with lipid-lowering therapy in hyperlipidemic patients. Pharmacoepidemiol Drug Saf 11(7): 593-600

15. Simon JA, Lin F, Hulley SB, Blanche PJ, Waters D, Shiboski S, et al. (2006) Phenotypic predictors of response to simvastatin therapy among African-Americans and Caucasians: the Cholesterol and Pharmacogenetics (CAP) study. Am J Cardiol 97(6):843-850

16. Wong MC, Jiang JY, Griffiths SM (2011) Adherence to lipidlowering agents among 11,042 patients in clinical practice. Int $\mathrm{J}$ Clin Pract 65(7):741-748

17. Sirey JA, Greenfield A, Weinberger MI, Bruce ML (2013) Medication beliefs and self-reported adherence among community-dwelling older adults. Clin Ther 35(2):153-160

18. DuMonthier WN, Haneline MT, Smith M (2009) Survey of health attitudes and behaviors of a chiropractic college population. $\mathrm{J}$ Manip Physiol Ther 32(6):477-484 